\title{
IMPACT OF THE TRANSPORT ON THE URBAN HEAT ISLAND
}

\author{
Haddad Louiza ${ }^{1}$, Aouachria Zéroual ${ }^{2}$, Haddad Djamel ${ }^{3}$ \\ ${ }^{1}$ Laboratory of Natural Risks and Regional Planning, University of Batna, Algeria \\ ${ }^{2}$ Laboratory of Applied Physics Energetic, University of Batna, Algeria \\ ${ }^{3}$ Hygiene and Security Institute, University of Batna, Algeria
}

Received 26 February 2015; accepted 20 July 2015

\begin{abstract}
Although transport has resulted in many beneficial effects on society, but their development in fact have negative impacts on the environment. The car policy caused many problems such as: - the spectacular growth of fuel consumption hence the very vast increase in urban pollution, traffic congestion in certain places and at certain times, the increase in the number of accidents. The exhaust emissions from cars and weather conditions are the main factors that determine the level of pollution in urban atmosphere. These conditions lead to the phenomenon of heat transfer and radiation occurring between the air and the soil surface. These exchanges give rise, in urban areas, to the effects of heat islands that correspond to the appearance of excess air temperature between the city and its surrounding space. We perform a numerical simulation of the plume generated by the exhaust gases of cars and show that these gases form a screening effect above the urban cite which cause the heat island in the presence of wind flow. The study allows us: $i$ - to understand the different mechanisms of interactions between these phenomenons, ii- to consider appropriate technical solutions to mitigate the effects of the heat island.
\end{abstract}

Keywords: atmospheric pollution, impact on the health, urban transport, heat island.

\section{Introduction}

Urbanization is one of the major events of the modern world due to its progress at a pace that tends to accelerate. Today there are more than 3 billion urban dwellers and, by 2025 , there could be three quarters of the population or even $80 \%$ of the world population living in urban cities (Rogers and Gumuchdjian, 2000). This urbanization causes an increased need for mobility in conjunction with the expansion of trade and the improvement of accessibility to different parts of the globe which then leads to congestion in traffic. Urban centres are often developed according to specific natural settings and thus in singular conditions. Thus, the transport will concentrate mainly in the urban areas and its impact will therefore appear dramatically on the environment and the atmosphere that lead to climate change. Cars and vehicles contribute to heat emission in urban areas. The total heat emitted by vehicles may remain trapped in poorly ventilated urban canyons, thereby reducing the thermal comfort of city dwellers. Vehicle emissions also contribute to the formation of urban smog and global warming (Wang et al., 2004; Watkins et al., 2007; Younger et al., 2008). Climatic conditions and urbanization have harmful effects on society directly

${ }^{1}$ Corresponding author: aouchria90@yahoo.fr 
through the most intense heat waves that can give rise to what was called the effect of urban heat island and the latter occurs because of urban microclimates and higher summer temperatures (Landsberg, 1981; Oke, 1982). According to observations, this temperature difference, mainly due to the urban built environment, varies between 2 and $12^{\circ} \mathrm{C}$ and is particularly threatening for the urban population (Oke, 1987; Voogt, 2002). The changing demands of summer comfort in buildings and air conditioning have vastly grown recently. Thermal loads could be doubled by the heat island effect, while the coefficient of performance
(COP) of air conditioning systems could be reduced by $25 \%$ (Martin and Maystre, 1988). The densification of cities and the overall increase in energy consumption cause the increase of the energy dissipated, and air conditioning systems represent a significant contribution as their load increases with warming. This allows us to schematize this issue in Fig. 1. There is also the phenomenon of convection: when the air is heated by the city it rises as it expands and becomes lighter than cold air. In short, it cools and falls. Thus creates a "dome" above the city where air masses move in an upward motion.

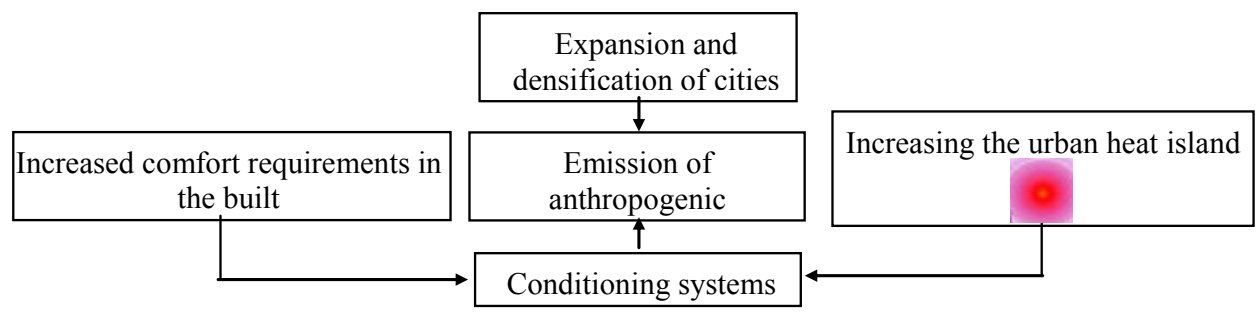

Fig. 1.

The Structure of the Interaction of Urban Densification and Comfort Requirements of the Building that Cause the Increase of the Effect of the UHI

\section{Urban Heat Island Phenomenon}

\subsection{What is an Urban Heat Island?}

An urban heat island is defined as an urbanized area characterized by higher summer temperatures than the surrounding environment (Escourrou, 1991). The discovery of this physical phenomenon dates back to 1833, when Howeard (1833) has undertaken a study of the temperature of the air in London and its surroundings (Fig. 2). The wind in the city is the result of the upward movement of warm air masses to the cold regions. In the urban context, where the heat differences are much more numerous, and cause the emergence of breezes (Fig. 2), that is to say weak winds turn cold areas to warmer areas. This is particularly important in the context of the urban heat island: this causes breezes from the countryside to the city, but even within the city, between, for example a park, colder and hot streets, or even within the same street. These breeze campaigns create a dome effect over the city and are particularly harmful during peak pollution. 


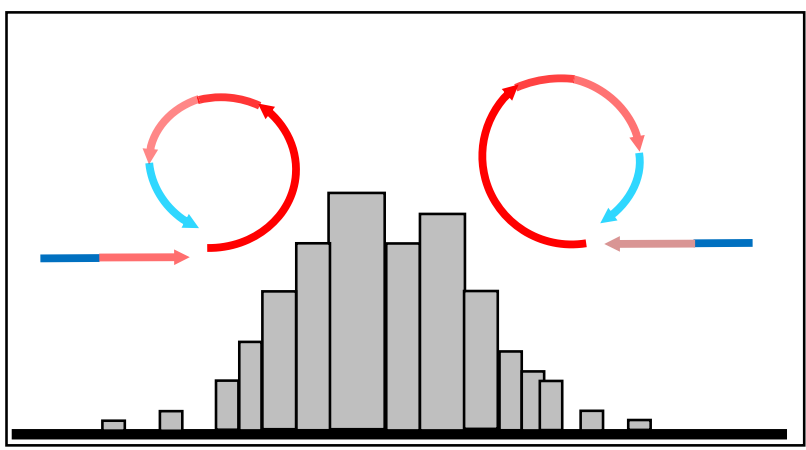

Fig. 2.

Thermal Breeze from Rural to the City

Typically this temperature excess is of about $\Delta \mathrm{T}=2-4^{\circ} \mathrm{C}$ which depends on the population and the wind speed in the form of Eq. (1) (Cappe, 2003):

$\Delta T_{(u-r) M A X}=\frac{P^{0.25}}{(4 U)^{0.5}}$
This excess varies depending on the size of the agglomeration (Fig. 3) (Martin and Maystre, 1988), season, location, time, and the speed of the wind (Table 1). These phenomena are associated with frames and more dense urban areas. They are caused by the absorption of solar energy by urban areas, mainly dark surfaces such as roofs and pavements. Part of this energy is reflected (Albedo).

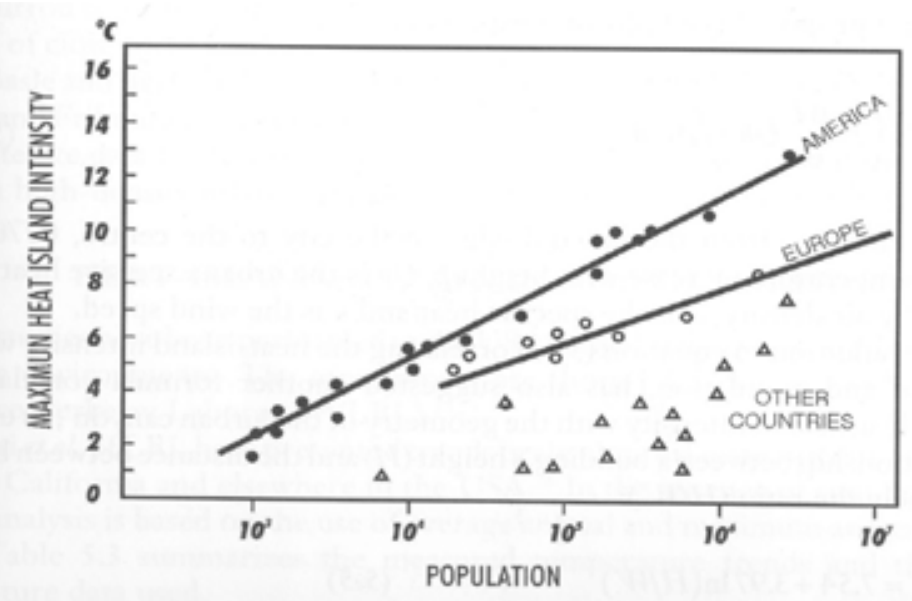

Fig. 3.

Evolution of the Maximum Intensity of the ICU 
Table 1

Intensity of ICU versus the Speed of the Wind in Paris City Center

\begin{tabular}{|l|l|}
\hline Wind speed $\mathbf{~ m} / \mathbf{s}$ & ICU Intensity \\
\hline 1 & 4.5 \\
\hline 2 & 3.4 \\
\hline 3 & 3.4 \\
\hline 4 & 2.6 \\
\hline 5 & 2.2 \\
\hline
\end{tabular}

\subsection{Factors Contributing to the Formation of Urban Heat Islands}

In urban areas, roofs, walls of buildings, are important factors in the formation of urban heat islands. Indeed, the fact of reduction of green space and changing it with materials that absorb heat promotes the formation of heat island since these materials once they have absorbed heat during the day to emit it overnight. Referring to Fig. 4, we find the Albedo values for different types of urban surfaces. Albedo measures the amount of solar energy reflected by a surface (Escourrou, 1991). Albedo is a measure of the ability of a surface to return the incident solar energy (which comes to the surface of the earth). It is a number between 0 and 1 ( 0 being a perfectly black surface which absorbs all the incident energy and 1 in perfect mirror that reflects all of the incident energy). A low Albedo means higher energy absorption and higher surface temperatures. Contrariwise, if the Albedo is high, it means cooler temperatures. In this case, we can see that the asphalt (Albedo 0.05-0.20) and tar and gravel (Albedo 0.3 to 0.18 ) are favorite places for urban heat islands. In addition, the black surfaces exposed to the sun can become hotter than the white surfaces, in order of $21^{\circ} \mathrm{C}$. We can then say that a potential method of reduction of heat island is to use high Albedo roofs for very dense cities, consisting of older and low-rise buildings in hot and sunny climates. With the high use of air conditioning, the presence of a heat flux related to district heating, traffic and industrial activity are also significant factors that contribute to the formation of urban heat islands.

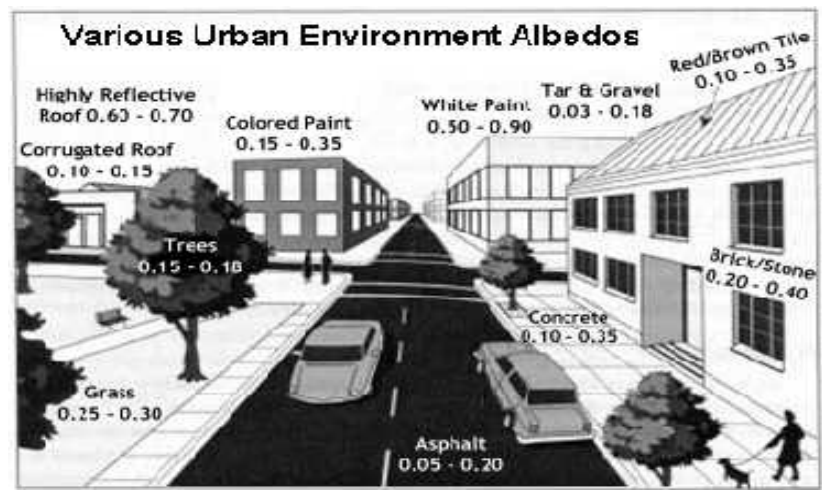

Fig. 4.

Albedo Values of an Urban Area 
Finally, the current climatic conditions experienced more frequent periods of extreme heat contributing to increases in minimum and maximum temperatures and an increased number of days of extreme heat once again favors the formation of urban heat islands (Fig. 5).

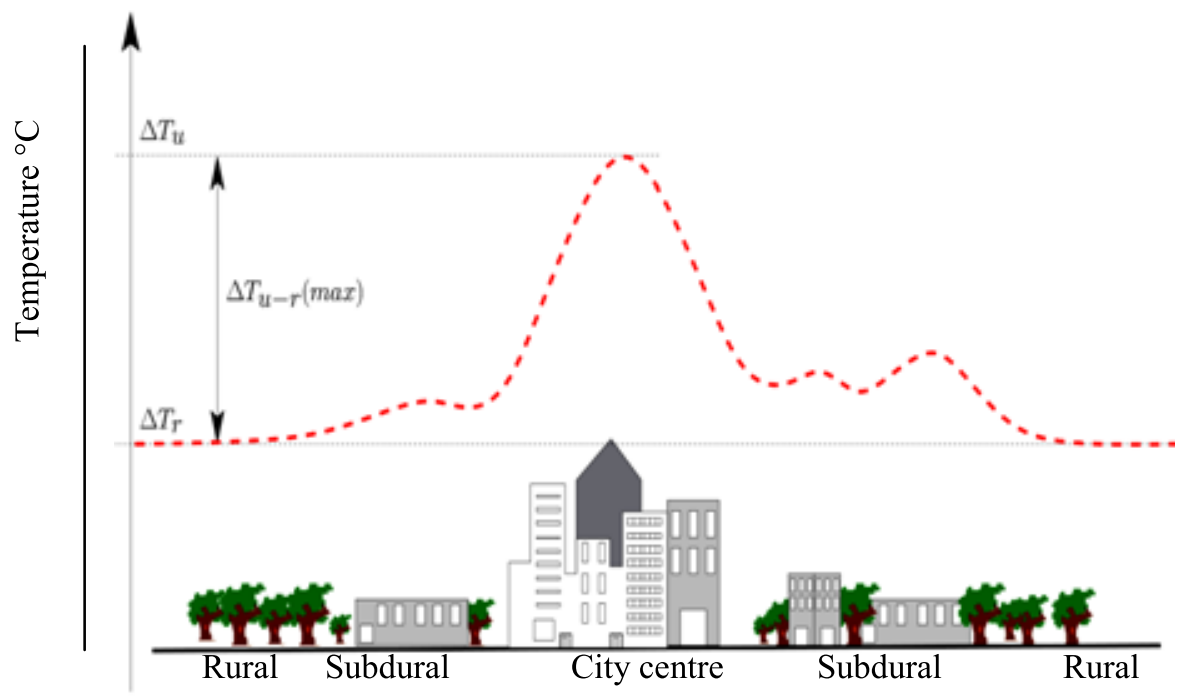

Fig. 5.

Thermal Profile of Urban Heat Island

\subsection{Consequences of Urban Heat Islands}

The urban heat islands increase electricity consumption for air conditioning. So it takes more energy to meet this need, which results in an increase in air pollution in the form of smog and sulfur dioxide and greenhouse gas emissions (Howeard, 1833). The urban heat island can make life difficult for people with respiratory illnesses. The dispersion of pollutants will be even stronger when the wind speed is high. The turbulence can be described to be as similar to the molecular motion occurring on a large scale movement. This phenomenon governing the exhaust gas of cars in urban areas is one of the most difficult issues to treat mathematically. Our study is an attempt which will focus on the contribution and influence of transport on the phenomenon of the urban heat island.

\section{Mathematical Formulation}

\subsection{Physical Analysis of the Phenomenon}

To answer the above questions, it is necessary to emphasize the differences between the city and the surroundings. Basically the city has a significant roughness forcing the air to rise and change the energy balance. A floor cover which inhibits the anthropogenic evaporation phenomenon leads to the production of energy emission of large amounts of pollutants. In the area of urbanization normal energy balance is written as Eq. (2): 
$\Phi^{*}=\left(\Phi_{\mathrm{D}+} \Phi_{d}\right)(1-a)+\Phi_{L}^{\downarrow}+\Phi_{L}^{\uparrow}$

Where $\Phi^{*}$ is the net energy received, $\Phi_{\mathrm{D}}$ is directly radiated solar energy, solar energy $\Phi_{d}$ is scattered by aerosols, $\Phi_{L}{ }^{\downarrow}$ and $\Phi_{L}^{\uparrow}$ are latent and sensible heat respectively. The infrared balance $\Phi_{L}^{\downarrow}+\Phi_{L}^{\uparrow}$ is averaged, in effect; the fraction $\Phi_{L}{ }^{\downarrow}$ of the radiation of the atmosphere tends to increase due to large number of aerosols that trap solar energy and converts it into infrared which is especially sensitive at night. This explains in part the island heat at night, despite the fact that infrared balance may change. To explain the presence of the urban heat island, we must take into account the energy received by the community. This consumption is as follows (Eq. (3)):

$\Phi^{*}+\Phi_{a}=\Phi_{H}+\Phi_{E}+\Phi_{S}$

where $\Phi_{a}$ is the production of human energy which is mainly due to industry and transportation, $\Phi_{H}$ is the sensible energy heating the lower layers of air, $\Phi_{E}$ is the latent energy carried by evapotranspiration and $\Phi_{S}$ is the building storage energy. To highlight the impact of transport on the heat island effect, we focus on the part of the energy generated by urban transport.

\subsection{Physical and Mathematical Modelling}

In the context of interest here, the development of these means of transport could reduce emissions of anthropogenic heat involved in the heat island effect, especially in the summer regarding the automobile (Fig. 6). We can approximate the gas transportation (including cars, for example) by panache of gas coming out of a chimney height (Fig. 6). The presence of significant concentrations of soot in these plumes also causes their heating by absorption of radiation, which contributes to their ascent. Mathematical modeling of fluid flow, which is actually a mathematical representation of the phenomenon involves determining at any point and at every moment the state variables which represent the fluid flow by solving a system 'partial differential equations. This system of equations governing the phenomenon of these emissions is derived from the application of conservation principles of mechanics and thermodynamique.
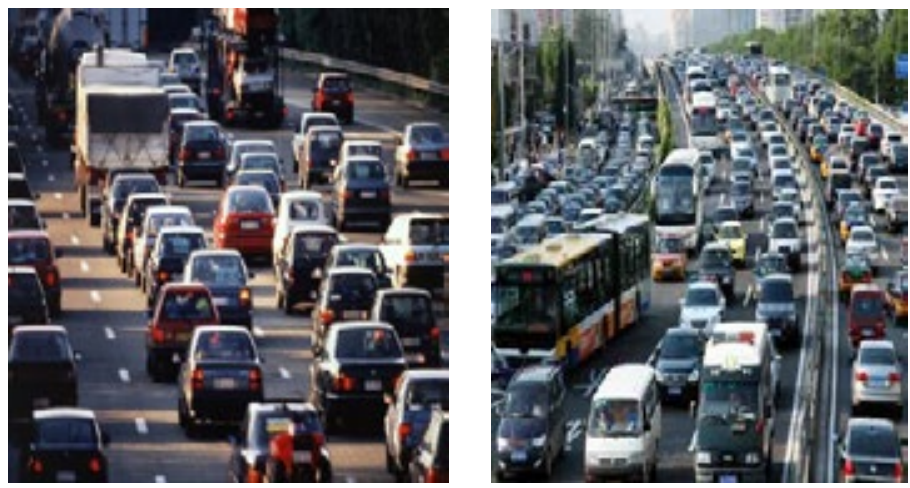

Fig. 6.

Urban Transport also Contributes to the Formation of Heat Islands 
Processes affecting pollutant concentrations during transport are complicated, often nonlinear, and cannot be investigated solely from the observations. It is therefore necessary to use numerical models to represent and assess their impact on the plumes. Auto emission is likened to a chimney that removes these air emissions (carbon dioxide and air in our case) with a speed $U_{0}$ and temperature $T_{0}$ and a fraction $Y_{0}$ of pollutant $\left(\mathrm{CO}_{2}\right)$ (Fig. 7a). A plume which is subjected to the wind velocity $U_{0}$ and temperature $T$ is obtained.
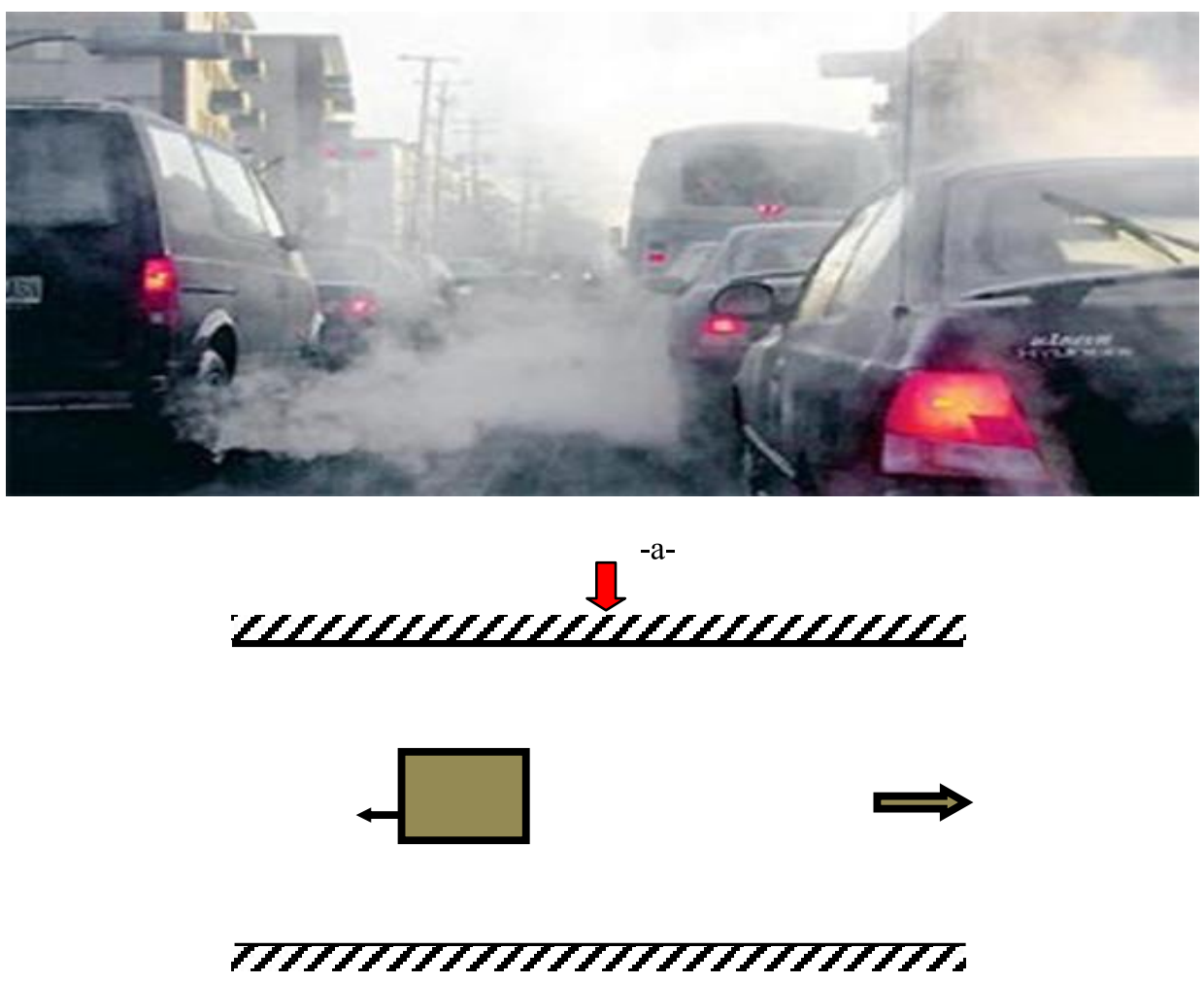

-b-

Fig. 7.

a-Gases Cars, b-Computation Model Area

Under the following conditions that the flow is considered two-dimensional, incompressible, obeying the Boussinesq approximation, and turbulent form, the wind speed is constant and the temperature gradient in the atmosphere is neglected. The study site and the reactive gas are in a rectangular area (Fig. $7 \mathrm{~b}$ ). The equations governing the flow of these emissions can therefore be written in the compact form as Eq. (4):

$\frac{\partial \rho \phi}{\partial t}+\sum_{j} \frac{\partial\left(\rho u_{i} \phi\right)}{\partial x_{i}}=\sum \frac{\partial}{\partial x_{i}}\left(\Gamma_{\phi} \frac{\partial \phi}{\partial x_{i}}\right)+S_{\phi}$ 
The first term at left represents the temporal variation of the quantity $(\rho \phi)$; the second term is the convected term and it represents the $(\phi)$ transported by the mass flow $\left(\rho u_{i}\right)$. The right terms represent respectively the diffusion and the source term. Although the behavior of some variables $\phi$ to be governed by the transport equation, mass flow should check the continuity equation that can be written as Eq. (5):

$$
\frac{\partial \rho}{\partial t}+\frac{\partial}{\partial x_{i}}\left(\rho u_{i}\right)=0
$$

The Eq. (4) can also be written in the form where we use the total flux (convective and diffusive) $J$ (Eq. (6)):

$$
\frac{\partial \rho \phi}{\partial t}+\sum_{j} \frac{\partial(J)}{\partial x_{i}}=S_{\phi}
$$

Where $J=\left(\rho u_{i} \phi-\Gamma_{\phi} \frac{\partial \phi}{\partial x_{i}}\right)$ and $S_{\phi}$ are the source term. The tree terms are given in Table 2, for each equation. Numerical simulation gives the results presented below in Table 2.

Table 2

Values of $\phi, \Gamma$ and $S_{\phi}$ in each Equation

\begin{tabular}{|l|l|l|l|}
\hline & $\phi$ & $\Gamma$ & $\mathrm{S} \phi$ \\
\hline Continuity equation & 1 & 0 & 0 \\
\hline X component of momentum equation & $\mathrm{U}^{*}$ & $\frac{1}{R e}$ & $-\frac{\partial P}{\partial x^{*}}$ \\
\hline Y component of momentum equation & $\mathrm{V}^{*}$ & $\frac{1}{\operatorname{Re}}$ & $-\frac{\partial P}{\partial x^{*}}+\theta$ \\
\hline Energy equation & $\theta$ & $\frac{1}{\operatorname{RePr}}$ & 0 \\
\hline Mass transport equation & $\mathrm{C}^{*}$ & $\frac{1}{\operatorname{ReSc}}$ & 0 \\
\hline
\end{tabular}

\section{Results and Discussion}

Fig. 8 shows the velocity vector field obtained at a given Reynolds number $\left(10^{5}\right)$ and at different time steps. We observe the velocity vector of the exhaust gases of a car is not influenced at all by the air velocity and is true only in the neighbouring hood of the exhaust exit. However, we notice some deviations at the velocity vector, which are generated downstream at the end of the exhaust. These deviations generate a turbulent vortex, which is amplified with time, acquiring kinetic energy, resulting in upward diffusion to the upper levels of the buildings in the neighbour hood, and even reaching the surrounding area. The presence of the vortex traps many particles thus leading to an accumulation of the pollutant near and on the soil. This result is in agreement with that reported by Aouachria and Haddad (2007) and Meguellati (2006). This result is illustrated in the Fig. 9 at different time steps $(t=100$, to $t=350)$. This accumulation is involved in the formation of heat islands. Fig. 9 shows the iso contours of the mass fraction of pollutantt. For a heterogeneous distribution of the latter we see that the area 
where this accumulation is important is in the recirculation zone corresponding to the maximum kinetic energy where it decreases moving upwards. We have represented in the Fig. 9 the evolution of the mass fraction of the pollutant with the time. We notice how well the deposition of pollutants, of burned gases, on the ground is increasing over time to cover the entire road floor. The deposition of pollutants changes the optical properties for levels surfaces that it covers such as the parameter of absorption of energy and its emission (Albedo). This phenomenon contributes to urban heat island formation. The propagation velocity of the mass of exhaust gas is large. It is sufficient to have a few minutes to smell. Indeed, we plotted the evolution diffusion of pollutants for four positions $(y=1, y=0.25, y=0.5, y=$ 0.75) in Fig. 10. The first thing that draws our attention is that the deviations of time because the pollutant, from one point to another is not the same although these positions are equally spaced. However, the spread of pollutants towards the last three positions is performed in a similar manner to a value of the nearby mass fraction of 0.65 around which it remains oscillate in all positions (Fig. 10). This explains the increase in temperature in this region than elsewhere. The hottest areas are located in the recirculation zone which is usually just above the centers of cities. This further magnifies their risk of pollution $\left(\mathrm{CO}_{2}\right)$ generated by the combustion of hydrocarbons, which participates in the formation of the greenhouse effect, especially when the wind speed is not zero (Hadef and Aouachria, 2014). In the case of a heat island, at ground level, the isotherms close around the city. Over time the temperature increases. When the wind is moderate, a phenomenon known as urban panache develops and defines the urban boundary layer. However it forms a cell which promotes the dome of pollutants and the stagnation over a city and a slow convective flow (Hadef et al., 2008).

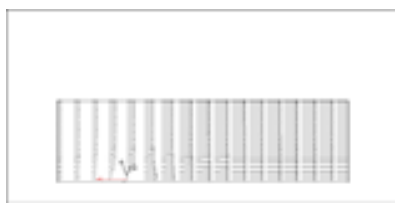

$\mathrm{t}=10$

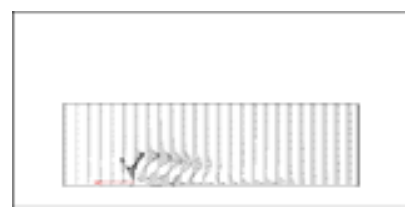

$\mathrm{t}=100$

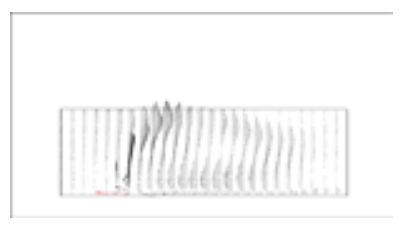

$t=300$

Fig. 8.

Evolution of the Dynamic Field in the Dimensionless Time 


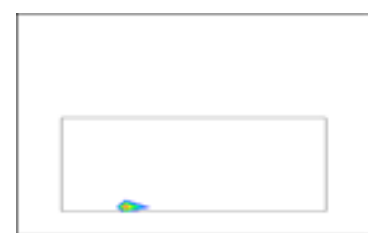

$\mathrm{t}=10$

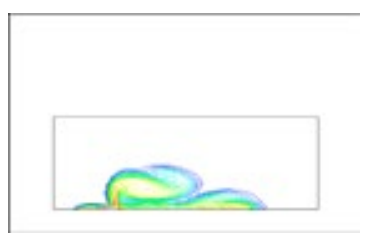

$t=100$

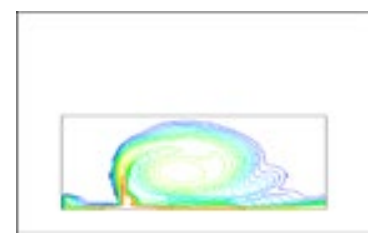

$\mathrm{t}=250$

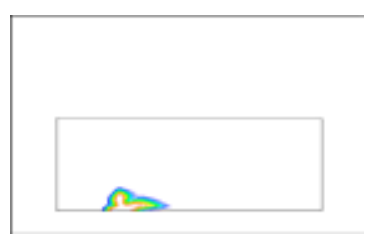

$t=25$

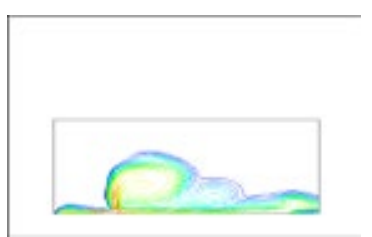

$\mathrm{t}=150$

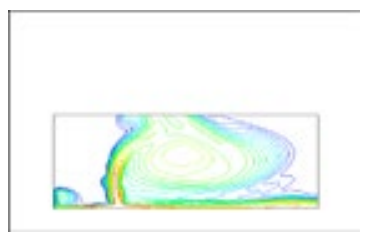

$t=300$

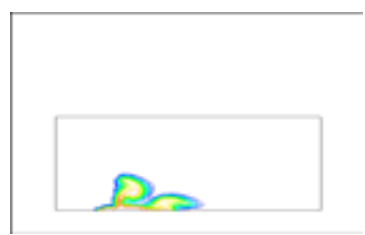

$\mathrm{t}=50$

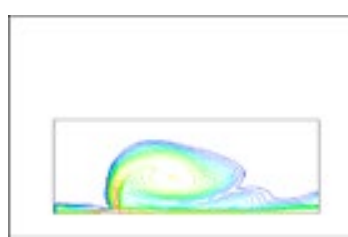

$t=200$

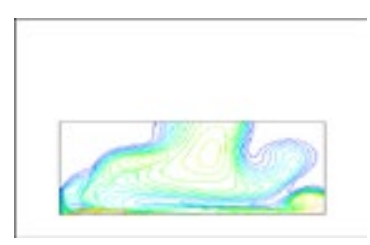

$t=350$

Fig. 9.

Evolution the Gase Fraction in the Dimensionless Time

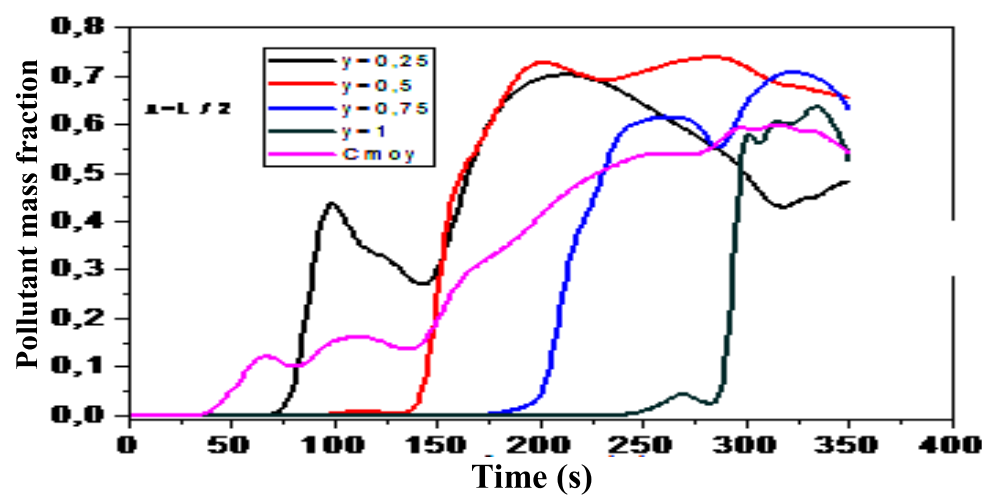

Fig. 10.

Evolution the Mass Fraction in the Dimensionless Time, for $x=L / 2$

To significantly reduce the impact of traffic, the objective is to seek to reduce the use of cars. And this can only be achieved through an approach to promote the use of active and public transport, while reducing the number of parking spaces, creating a pedestrian environment, and making the vegetation of the city. Two factors contribute to the raising of a plume emitted from a point source (exhaust): i- the strength of 
convective flow; ii- buoyancy due to heat plume relative to middle ambient.

\section{Conclusion}

The ICU is an urban effect given consideration in the design and management of the city. As the phenomena of ICU, climate change and air pollution are interrelated ways to implement a fight against these problem. It is therefore essential to work towards reducing the emissions potentially toxic to humans and the environment, ejected by the major sources recognized as urban transport. In this context, we consider the industrial use of hydrogen as a fuel to replace fossil hydrocarbons. Indeed, the gases produced by combustion contain no $\mathrm{CO}_{2}$ or $\mathrm{CO}$ or soot and unburned hydrocarbons. The fight against the ICU also requires a reassessment of planning policies and implies preference of white or colored surfaces, reforesting cities, developing transport, and real changes in the behavior of future users of transportation which will not be achieved through an approach to promote the use of active and collective transportation. This study confirm that transport constitute one of principal specific factors to the urban heat island increase in city, the raison that let transport considered as a stake in intervention on heat island reduction to develop a proper durable city. To achieve this purpose, some measures are recommended: 1. Encouragement of public transport: to reduce fuel consummation and gas emission in the city, the encouragement and motivation of public transport by decreasing the tariff of ride public transport is necessary; 2. Insist on using proper transports: walking and displacement by bike are the most economic and proper modes of transport; 3. Amelioration of fuel quality: actually, several methods to ameliorate fuel quality are available for decreasing gas emission. However, these methods are few spreading especially in third world country; 4. Vegetalisation of city: vegetation in the city is urban furniture that contributes to the urban atmospheric equilibrium by the absorption of gaseous pollutants and their air warming braking. It is therefore appropriate to encourage throughout the city; 5. Displacement economization: with the development of the means of telecommunication, several displacements by car will void if taking advantage of these means in the district of interests. This matter allows the diminution of particular vehicles and the reduction gaseous emissions of pollutants.

\section{References}

Aouachria, Z.; Haddad, L. 2007. Dispersion of a pollutant in air flow along a channel, Asian Journal of Technology, 6(2): 181-184.

Cappe, M. 2003. Péage urbain: l'exemple de Londres en Angleterre (in French). Infrastructures, Canada, Ottawa, $35 \mathrm{p}$.

Escourrou, G. 1991. Le climatet la ville (in French). Presses universitaires Nathan, Paris.

Hadef, A.; Aouachria, Z. 2014. The interactions of the kinetic chemistry and the turbulence on the turbulent diffusion flame. In Proceedings of the AIP Conference. DOI: http://dx.doi.org/10.1063/1.4823898, 1557: 174-179.

Hadef, A.; Aouachria, Z.; Mamerie, A. 2008. Modélisation d'une Flamme Turbulente de Diffusion (H2-Air) par une Approche Statistique (in French), JM08, ENITA, Algérie.

Howeard, L. 1833. The climate of London deduced from; meteorological observations. 3rd Edition in 3 volumes, London. 
Landsberg, H.T. 1981. The urban climate. New York: Academic Press, 275 p.

Martin, J.; Maystre, L.Y. 1988. Santéet pollution del'air (in French). Presses polytechniques romandes, Lausanne, $146 \mathrm{p}$.

Oke, T.R. 1987. Boundary layer climates. 2nd ed., Route ledge, London, $474 \mathrm{p}$.

Oke, T.R. 1982. The energetic basis of the urban heat island, Quarterly Journal of the Royal Meteorological Society. DOI: http://dx.doi.org/10.1002/qj.49710845502, 108(455): 1-24.

Rogers, R.; Gumuchdjian, P. 2000. Des Villes pour une petite planète (in French). Moniteur, Paris, $22 \mathrm{p}$.

Voogt, J.A. 2002. Urban heat island, Encyclopaedia of global environmental change, 3: 660-666.
Wang, G.; Macera, C.A.; Scudder-Soucie, B.; Schmid, T.; Pratt, M.; Buchner, D.; Heath, G. 2004. Cost analysis of the built environment: the case of bike and pedestrian trails in Lincoln Neb, American Journal of Public Health, 94(4): 549-553.

Watkins, R.; Palmer, J.; Kolokotroni, M. 2007. Increased temperature and intensification of the urban heat island: implications for human comfort and urban design, Built Environment, 33(1): 85-96.

Younger, M.; Morrow-Almeida, H.R.; Vindigni, S.M.; Dannenberg, A.L.; 2008. Built environment, climate change, and health: opportunities for co-benefits, American Journal of Preventive Medicine. DOI: http://dx.doi. org/10.1016/j.amepre.2008.08.017, 35(5): 517-526. 